\title{
Photometric variability of southern T Tauri stars ${ }^{\star, \star \star}$
}

\author{
C.C. Batalha ${ }^{1}$, G.R. Quast ${ }^{2}$, C.A.O. Torres ${ }^{2}$, P.C.R. Pereira ${ }^{3}$, M.A.O. Terra ${ }^{1}$, F. Jablonski ${ }^{3}$, \\ R.P. Schiavon ${ }^{4}$, J.R. de la Reza ${ }^{1}$, and M.J. Sartori ${ }^{4}$ \\ 1 Observatório Nacional/CNPq/MCT Rua Gal. José Cristino, 77, 20921-400 Rio de Janeiro, RJ, Brazil \\ 2 Laboratório Nacional de Astrofísica/CNPq/MCT Rua Estados Unidos, 154, CP 21, 37500-000 Itajubá, MG, Brazil \\ 3 Divisão de Astrofísica - INPE/MCT, CP 515, 12201-970 São José dos Campos, SP, Brazil \\ ${ }^{4}$ Instituto Astronômico e Geofísico - USP, CP 9638, 01065-970 São Paulo, SP, Brazil
}

Received June 23; accepted September 1, 1997

\begin{abstract}
We present photometric light curves of T Tauri Stars (TTS) observed in the Southern hemisphere. Our list includes 26 TTS, half of which were discovered by the Pico dos Dias survey (PDS). The majority of the observed PDS stars are weak TTS and present, on average, the low range of variability typical of such stars. We monitored some of the stars for several seasons in order to detect possible changes in rotational modulation. In particular, we find that the classical TTS, AS 216, shows a stable period of $3.12 \mathrm{~d}$ for three consecutive years (between 1985 and 1987) which then does not reveal itself during the observing season of 1989 and 1990. Coupled with this change during the last two observing seasons is an overall increase in the amplitude of the photometric variability in all colors. We derive photometric periods for 13 previously unmonitored objects, and confirm the previously published rotational period of SY Cha. In addition, we confirm that TW Cha and SZ 82 do not reveal any signs of periodic modulation.
\end{abstract}

Key words: stars: pre-main sequence - stars: variable

\section{Introduction}

The work of Joy (1942) identified T Tauri Stars (TTS) as a new class of stars characterized by irregular photometric variability. Several TTS became targets of constant photometric observation - some even subject to systematic daily monitoring during the 80's. Herbst et al. (1994) combined

Send offprint requests to: Celso Batalha celso@on.br

* Based on observations made at Laboratório Nacional de Astrofísica/CNPq/MCT, Brazil.

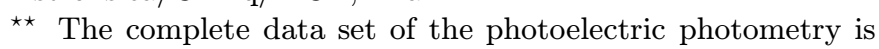
only available in electronic form at the CDS, via anonymous ftp to cdsarc.u-strasbg.fr (130.79.128.5) or via http://cdsweb.ustrasbg.fr/Abstract.html photometry from the literature and established periodicity for a number of these low mass pre-main sequence stars. Several stars show irregular variations superimposed on the periodic patterns. TTS with large accretion rates present, in general, no detectable periodic behavior (see Gahm et al. 1993; Bouvier et al. 1993; Covino et al. 1992).

It is often assumed that a classical TTS (cTTS) possesses a dipole magnetic field that disrupts the geometrically thin circumstellar disk, funneling the incoming gas onto high latitude regions of the central star, thereby generating hot spots. Theory (Königl 1991; Shu et al. 1994), observations (Johns \& Basri 1995; Kenyon et al. 1994; Edwards et al. 1994) and semi-empirical models (Hartmann et al. 1994) support this scenario in which hot spots covering a total projected area of less than $3 \%$, are sitting at the base of the magnetic column. If the field is axially symmetric over the stellar surface and not aligned with the geometric poles, then the light curve will modulate with the same period of the stellar rotation. In fact, the comprehensive catalogue of Herbst et al. (1994) indicates that high latitude hot spots account for the light modulation of classical TTS (cTTS), whereas cool spots are responsible for that of weak TTS (wTTS).

Bouvier et al. (1993) highlight two important findings in their photometric campaign of the Taurus - Aurigae complexes. First, they trace periodic light curves for all of the objects in their sample (24 stars). After comparing the equatorial velocity inferred from the computed periods with the projected vsini, they conclude that the transit of surface spots are the most likely source of light modulation observed in 20 of their stars ( $88 \%$ of the sample). A second important finding emerges after including an additional sample of 17 TTS with previously established periods in their data set. They conclude that the TTS rotational periods segregate themselves into two distributions with a mean value of 4.1 days for wTTS and $7.6 \mathrm{~d}$ for cTTS. Almost simultaneously, the analysis of previously published rotational periods leads Edwards et al. (1993) to similar conclusions, with a somewhat larger spread in 
the distribution of periods for the wTTS. The recent campaigns of Bouvier et al. (1995 and 1997) confirm these findings.

Why, on average, do wTTS rotate faster than the cTTS? Both weak and cTTS undergo gravitational contraction with the resulting increase in their rotation rates. Furthermore, classical TTS have a surplus of angular momentum resulting from the torque applied to the stellar surface by the accretion disk which should therefore lower the periods. In addition to a magnetized stellar wind which would steadily deplete the stellar angular momentum, Edwards et al. (1993) propose an active coupling between the stellar magnetosphere and the slowly rotating outer regions of the disk as a possible solution to this dilemma. Thus, the stellar angular velocities of the cTTS would be kept at values that are, on average, lower than those found in wTTS.

Another view is championed by Smith (1994) and stems from the fact that differential rotation is established in the Sun but is conveniently ignored throughout the calculations of TTS periods which are computed assuming that the star rotates as a rigid body. Smith proposes that the bimodal distribution of rotational periods among classical and weak TTS is an artifact of the differential rotation. The classical TTS apparently rotate slower than the weak TTS because the spots that define their light curves are thought to be at high latitudes. On the contrary, those of the weak TTS are hypothesized to be at low latitude regions as in the solar analogy, although recent Doppler images of weak TTS consistently show high latitude features (Joncour et al. 1994; Strassmeier et al. 1994). The question remains whether the bimodal distribution of periods is an indication that steep differential rotation prevails among PMS stars or whether the stellar initial angular momentum is controlled by the presence of an accretion disk.

These competing interpretations can only be settled with systematic photometric and/or high resolution spectroscopic monitoring campaigns. It is crucial to devote observational efforts in order to increase the statistics of rotational periods among TTS. Monitoring selected targets over several consecutive seasons, tracking possible changes from periodic to aperiodic patterns, and ideally combining with spectroscopic timeseries and Doppler imaging, may shed some light on the nature of these pre main sequence objects - their level of stellar activity, their initial angular momentum and their angular momentum evolution.

The main goal of this work is to increase the information about the photometric variability of $\mathrm{T}$ Tauri Stars. It is based on photometric data collected by different researchers during the past 10 years at Pico dos Dias Observatory (OPD), including extensive photoelectric monitoring of AS 216, AS 218 and FK Ser. In Sect. 2 we describe the observations and data reduction. The periodogram methods employed in this investigation are described in Sect. 3 and results are summarized in Sect. 4.

\section{Observations and data reduction}

CCD photometry was carried out from 1993 to 1995 at the $0.6 \mathrm{~m}$ telescopes of OPD with a front illuminated GEC P8603A CCD detector with resolution of about $0.58^{\prime \prime} /$ pixel $(385 \times 578$ pixels $)$. No attempt was made to transform the computed magnitudes to a standard system since our main interest is to study time variations. This procedure does not require photometric sky conditions and successfully overcomes the problematic effects of variable transparency. In deciding between observing a large sample of diverse objects or fewer objects at more colors, we chose the former. Therefore, our images were taken with the $V$ and $I$ filters, and exposures were all 3 minutes long.

Photoelectric differential photometry in $U B V(R I)_{\mathrm{C}}$ were also taken with the same telescopes using the FOTRAP (Jablonski et al. 1994). This sample includes recently identified TTS sorted by their IRAS colors and conspicuous Li lines (Pico dos Dias Survey - henceforth PDS; Gregorio-Hetem et al. 1992, henceforth GHETAL92). Special attention is given to AS 216 and AS 218, monitored since 1985 to establish the stability of their rotational periods.

In general the objects are brighter than $V \sim 14.0$ and cooler than K5. T Cha (G8) is included because of its reportedly extreme variability.

\subsection{CCD photometry}

We adopt the standard method to perform the differential photometry. The stars in each field - excluding the variable target - are examined for stability and those showing no trace of variability are chosen as comparisons. The uncertainty in the photometry is estimated from the dispersion in the magnitude differences and ranges from 0.01 to 0.02 mag. The TTS observed with the CCD are SY Cha, TW Cha, CT Cha, VZ Cha, WY Cha, SZ 45, T Cha, SZ 77, SZ 82 and SZ 108.

Data reduction is carried out with the Image Reduction and Analysis Facility (IRAF) software. Images are corrected for electronic bias, pixel-to-pixel gain variations (with dome flats), and cosmic rays are removed. The routines of the APPHOT package are used to obtain relative magnitudes for the objects selected in each field. The sky contribution is the modal value inside a specified annulus whose internal radius and width are automatically determined for each image by the RADPROF routine. This procedure avoids possible effects of nearby stars or defective pixels. Typical values of the aperture range from four to seven pixels, corresponding to $2.3^{\prime \prime}$ to $4^{\prime \prime}$ respectively.

\subsection{Photoelectric photometry}

Photoelectric photometry was performed using the FOTRAP (Jablonski et al. 1994). This device has a 
Table 1. Summary of the observed Southern T Tauri Stars. The star names are listed in the first and second columns. The seasons are indicated in the third column. Columns 4 through 8 list the $\sigma$ of the differential magnitudes. The number of observations in each season are listed in Col. 9. The last column contains spectral types

\begin{tabular}{|c|c|c|c|c|c|c|c|c|c|}
\hline Star & Other name & Year & $\sigma U$ & $\sigma B$ & $\sigma V$ & $\sigma R$ & $\sigma I$ & NP & SpT \\
\hline PDS 01 & Hen 1 & 1989 & 0.01 & 0.02 & 0.03 & 0.03 & 0.02 & 4 & $\mathrm{~K} 1$ \\
\hline 45 & CoD-29 8887 & $1989 / 90$ & 0.15 & 0.06 & 0.04 & 0.04 & 0.04 & 10 & M3 \\
\hline 50 & Hen 600 & $1989 / 90$ & 0.17 & 0.05 & 0.02 & 0.02 & 0.02 & 13 & M3+M3 \\
\hline 59 & GSG 9419-1065 & 1991 & 0.34 & 0.11 & 0.11 & 0.10 & 0.07 & 9 & M1 \\
\hline 66 & Hen 892 & 1990 & 0.12 & 0.05 & 0.03 & 0.03 & 0.02 & 10 & $\mathrm{~K} 2$ \\
\hline 70 & CoD-40 8434 & 1990 & 0.06 & 0.04 & 0.03 & 0.03 & 0.02 & 9 & K7 \\
\hline 77 & CoD-41 10484 & 1990 & 0.47 & 0.43 & 0.37 & 0.33 & 0.29 & 7 & K0 \\
\hline 81 & GSC 6209-923 & $1989 / 90$ & 0.05 & 0.05 & 0.04 & 0.04 & 0.03 & 11 & $\mathrm{~K} 0$ \\
\hline 82 & VV Sco & 1990 & 0.17 & 0.15 & 0.10 & 0.08 & 0.06 & 7 & $\mathrm{~K} 0+\mathrm{M} 1$ \\
\hline 83 & V896 Sco & 1990 & 0.35 & 0.24 & 0.19 & 0.15 & 0.12 & 7 & K5 \\
\hline 89 & GSC $6217-126$ & 1990 & 0.12 & 0.08 & 0.07 & 0.06 & 0.04 & 4 & M0 \\
\hline 99 & & 1989 & 0.31 & 0.19 & 0.16 & 0.14 & 0.13 & 4 & M0 \\
\hline 101 & BZ Sgr & 1989 & 0.91 & 0.66 & 0.53 & 0.42 & 0.34 & 7 & K0 \\
\hline HBC 565 & SY Cha & $1994 / 1995$ & - & - & 0.23 & - & 0.14 & 22 & M0 \\
\hline 567 & TW Cha & $1994 / 1995$ & - & - & 0.29 & - & 0.14 & 18 & M0 \\
\hline 570 & CT Cha & 1995 & - & - & 0.08 & - & 0.04 & 10 & M0 \\
\hline 578 & VZ Cha & $1994 / 1995$ & - & - & 0.46 & - & 0.23 & 15 & K6 \\
\hline 583 & WY Cha & $1994 / 1995$ & - & - & 0.08 & - & 0.07 & 11 & $\mathrm{~K} 7$ \\
\hline 590 & $\mathrm{Sz} 45$ & $1994 / 1995$ & - & - & 0.15 & - & 0.05 & 10 & M0.5 \\
\hline 591 & T Cha & 1995 & - & - & 0.90 & - & 0.67 & 7 & G8 \\
\hline 603 & Sz 77 & $1994 / 1995$ & - & - & 0.13 & - & 0.08 & 10 & M0 \\
\hline 605 & Sz 82 & 1995/1994 & - & - & 0.07 & - & 0.05 & 16 & M0 \\
\hline 620 & Sz 108 & $1994 / 1995$ & - & - & 0.04 & - & 0.06 & 10 & M0 \\
\hline \multirow[t]{5}{*}{656} & AS 216 & 1985 & 0.21 & 0.09 & 0.05 & - & - & 21 & $\mathrm{~K} 2$ \\
\hline & & 1986 & 0.24 & 0.11 & 0.06 & - & - & 67 & \\
\hline & & 1987 & 0.23 & 0.09 & 0.05 & - & - & 41 & \\
\hline & & 1989 & 0.44 & 0.14 & 0.08 & 0.05 & 0.04 & 7 & \\
\hline & & 1990 & 0.45 & 0.16 & 0.09 & 0.07 & 0.06 & 11 & \\
\hline \multirow[t]{3}{*}{657} & AS 218 & 1986 & 0.93 & 0.30 & 0.21 & & & 56 & $\mathrm{~K} 7$ \\
\hline & & 1989 & 1.47 & 0.70 & 0.40 & 0.24 & 0.12 & 5 & \\
\hline & & 1990 & 0.49 & 0.13 & 0.08 & 0.07 & 0.07 & 11 & \\
\hline \multirow[t]{2}{*}{663} & FK Ser & 1986 & 0.89 & 0.32 & 0.18 & & & 30 & K5/K7 \\
\hline & & $1989 / 1990$ & 0.60 & 0.33 & 0.31 & 0.31 & 0.24 & 11 & \\
\hline
\end{tabular}

rotating filter wheel $(1200 \mathrm{rpm})$ that enables measurements in 5 colors in a quasi-simultaneous way. The photometric system is matched to the Johnson/Cousins $U B V(R I)_{\mathrm{C}}$ standard system. We achieve differential magnitudes by observing the stars in the order: TTS, comparison 1, comparison 2 and TTS. The two arrays of differential values per season (TTS - Comp) are then combined and used as input to the periodogram analysis. The final set of observed TTS include all the listed PDS objects and AS 216, AS 218, FK Ser.

Table 1 summarizes our observation logs. The first and second columns list the names of the objects and are followed by the year of the observation. Columns 4 through 8 list the $\sigma$ of the differential magnitudes. The number of observations in each of the observed seasons are indicated next. The spectral types are taken from the Herbig \& Bell catalogue (1988 - henceforth HBC) or from a spectral type classification method developed to utilize the medium resolution data of the PDS survey. This method essentially compares the unknown TTS spectrum to those of standards of different spectral class observed with the same instrument and spectral format. The spectral type of $\mathrm{T}$ Cha is taken from Alcalá et al. (1993). Complete electronic tables with the photoelectric magnitudes can be accessed at the CDS, via anonymous ftp to cdsarc.u-strasbg.fr or via http://cdsweb.u-strasbg.fr/Abstract.html.

\subsection{Periodogram analysis}

For all the stars but Hen 1, V896 Sco and PDS 89, we gathered enough observations to search for photometric periodicity. Since the observations were generally taken near meridian transit, our frequency search is limited to 
the interval 0.0 to 0.5 day $^{-1}$, the quasi-Nyquist frequency. We cannot discriminate these from the aliases which occur in the frequency interval between 0.5 and 1.0 day $^{-1}$.

We use the two following methods to access frequencies:

1) The Date Compensated Discrete Fourier Transform (DCDFT, Ferraz-Mello 1981) that computes the correlation between a sinusoid-plus-constant curve with the photometric data. The significance of the candidate peak is assessed with the method outlined by Ferraz Mello \& Quast (1987), and gives the probability that a peak in the distribution is not formed by chance (the acceptance of a given period). The method also gives the error bar of the computed period.

2) The method developed by Akerlof et al. (1994, henceforth BSP) that computes the least-square fit of a sum of cubic B-spline functions to the period-folded light curve. The chosen frequency is that which provides the minimum $\chi^{2}$ statistic. A characteristic of this method is that the computed frequency is not affected by any predefined light curve shape (e.g. sinusoidal). This degree of freedom allows a range of possible solutions for time series with less than 10 entries. We tested the efficiency of this program using the photometry of several TTS available in electronic format (Herbst et al. 1994). The program provides firm solutions for stars with large numbers of entries (though sometimes disagreeing with the published value) and handles data sets with fewer entries (down to 7), though not claiming a unique solution for those cases. The final range of solutions can be narrowed down to a few options if the shape of the folded light curve is fixed to a sinusoidal one.

Our basic procedure which leads to a final rotational period is as follows. First, we utilize the BSP code for the $V, R$ and $I$ colors and select the 15 frequencies, in each color, yielding light curves of low $\chi^{2}$. The final solution tends to be unique if the inputed time series has a large number of entries. In this case, that frequency is easily identified in every color and distinguishable from the others by its low $\chi^{2}$ (see example in Akerlof et al. 1994). In some cases, we have a modest data set for which, as discussed above, the BSP code ascribes several possible solutions. In these cases, we select as the final BSP solutions those where the shape of the $U, B, V, R$ and $I$ folded light curves are correlated in phase. In addition, the curve shall present an even distribution of data-points in phase. In some cases, we are satisfied with more than one $f_{-}$nal BSP solution. Secondly, we run the DCDFT code and search for the best frequency along the steps indicated by this method. We analyze individual color bands, selecting the solutions yielding good phase coverage, large acceptance in all colors ( $U$ excluded), and consistency among the period-folded light curves for each color band. The chosen DCDFT period is then compared with that of the BSP method and usually the sinusoidal-light curve solution of the former method is adopted.

\section{Photometric variability}

The largest photometric campaign of Southern TTS is presented by Covino et al. (1992), after monitoring a sample of 30 stars in $U B V(R I)_{\mathrm{C}}$. They confirm the previously reported period of $\mathrm{CV}$ Cha, $\mathrm{Sz} 6$ and $\mathrm{Sz} 68$ and establish new periods for four other stars (AS 205, Wa Oph/2, WA $\mathrm{Oph} / 3$ and Wa CrA/2). They are unable to confirm previously reported periods for $\mathrm{T}$ Cha and $\mathrm{S}$ CrA or to find any periodical behavior for SZ Cha and GQ Lup.

We analyze the light curves of the $26 \mathrm{~T}$ Tauri stars listed in Table 1 where the first 13 have only recently been identified as PMS stars.

Table 2 shows the periods determined in this work and the previously published ones. We label each star in Table 2 as weak or classical following the earlier criteria based solely on the strength of the $\mathrm{H} \alpha$ emission: wTTS if $\mathrm{EW}_{\mathrm{H} \alpha}$ is less than $10 \AA$ and cTTS if larger. However, the most complete set of criteria to distinguish cTTS from wTTS is discussed by Edwards et al. (1993). These are the near infrared color excess, the optical continuum veiling and the forbidden emission line of $[\mathrm{OI}]$ at $\lambda$ 6300. Such data are presently lacking for the majority of our targets.

The $\mathrm{EW}_{\mathrm{H} \alpha}$ are taken from the $\mathrm{HBC}$ or PDS. For a low mass PMS star, $\mathrm{H} \alpha$ line strength indicates (but not conclusively) the presence of a circumstellar disk since important components of this line are formed either in a wind or in an accretion column - both scenarios requiring disks (Johns \& Basri 1995). Thus, the $\mathrm{H} \alpha$ of Hen 892 and BZ Sgr suggest the presence of a disk. All the remaining PDS stars have small but overt $\mathrm{EW}_{\mathrm{H} \alpha}$. Further observations of these wTTS must be carried out, especially in the near infrared, in order to constrain their circumstellar environments.

\subsection{Stars with no firm detection of periodicity}

\subsubsection{PDS 1 (Hen 1), PDS 89 and PDS 99}

These objects were not observed with enough regularity to enable time series analysis. There are only 4 observations per star.

Hen 1 is not an IRAS source and does not indicate near infrared excess of luminosity (GHETAL92). It is situated at high galactic latitude $\left(b \sim-59^{\circ}\right)$ and is not associated with any known molecular cloud. The level of variability (as deduced solely from our 4 observations) is one of the lowest in our sample - at a level which is unusual for preMain Sequence stars.

PDS 89 is a serendipitous discovery of PDS since it lies outside of the error box of an IRAS source. The source is actually associated with PDS 149 in the small cloud L152 where HBC 651 is located (Torres et al. 1995). PDS 99 belongs to the $\mathrm{CrA}$ association and was previously identified as an $\mathrm{H} \alpha$ emission line star by Marraco \& Rydgren (1981). 


\subsubsection{PDS 45 (CoD-29 8887) and PDS 50 (Hen 600)}

These two PDS stars together with PDS 54, PDS 55 and the cTTS TW Hya lie within a circle of about $7^{\circ}$ at high galactic latitude $\left(b \sim 25^{\circ}\right)$. de la Reza et al. (1989) attempt to physically connect these objects as members of a common parental cloud which has already dissipated. Jensen et al. (1996) did not detect PDS 45 nor Hen 600 at $800 \mu \mathrm{m}$ but did detect PDS 55, which curiously is not an IRAS source. We have less than 4 entries for PDS 54 and PDS 55, and they are not included in this work.

Our photometry in the $V$-band of PDS 45 and 50 shows the low range of variability typically ascribed to solar-type active regions on the stellar surface (see Rodonò 1986). The amplitude of the variations reaches merely a few times the intrinsic errors in our measurements thereby preventing us from establishing a reliable rotational period.

\subsection{3. $\operatorname{PDS} 77=$ CoD-41 10484}

PDS 77 has the spectroscopic characteristics of a wTTS and is a member of the Lupus association. If the strength of $\mathrm{EW}_{\mathrm{H} \alpha}$ alone is the indicator of a circumstellar disk then PDS 77 is a wTTS and probably devoid of an active disk. However, Herbst et al. (1994) argue that wTTS generally have light fluctuations less than $0.8 \mathrm{mag}$ in $V$. Only accretion disks are capable of powering light variations that are larger than this value in the $V$-band.

We have gathered a sparse and small number of data points (7). Nevertheless, we observed a significant range of variability in all colors $(0.91$ at $V)$. Several possibilities are indicated by the DCDFT code and they all cluster about 2.50 days with mild acceptance. We suggest this as a preliminary result and we stress that additional observations are needed to confirm this.

\subsubsection{PDS 81 and PDS $82=\mathrm{VV}$ Sco}

These two stars are found in the same region of the $\rho$ Oph complex. PDS 81 presents a range of variability of $0.15 \mathrm{mag}$ in all colors. It is a wTTS with $\mathrm{H} \alpha$ sometimes filled in. It is associated with the cloud B40 for which HBC lists 5 TTS, and the PDS finds another 3. A total of 11 exposures in $U B V(R I)_{\mathrm{C}}$ were gathered in $1988 / 1989$ $(4 / 7)$ and the BSP algorithm does not indicate any reliable period. The DCDFT method indicates a period of 11.11 days but with a modest degree of confidence $(62 \%$ in $V)$ which leads us to not include it in the pool of TTS with determined rotational periods.

VV Sco is a binary system with a separation of $1.5^{\prime \prime}$. It lies at the border of the complex and cannot be resolved photometrically. We search for periodical patterns in the light curve with no conclusive results.

\subsubsection{PDS $101=\mathrm{BZ}$ Sgr}

The PMS nature of BZ Sgr was established quite recently (GHETAL92) but its $\mathrm{H} \alpha$ profile in emission had been known since earlier prism surveys (Stephenson \& Sanduleak 1977). GHETAL92 associated this object with the molecular cloud No. 159 (Magnani et al. 1985). We discover a very faint companion less than $5^{\prime \prime}$ apart. This possible companion has a firm emission in $\mathrm{H} \alpha$ but the noise prevents any conclusion as to the existence of the Li I line.

This star presents remarkable variability and striking color-dependent amplitudes that are typical of cTTS. The strength of its $\mathrm{H} \alpha$ also leads us to believe that this object is a classical TTS. Jensen et al. (1996) detect the star at $800 \mu \mathrm{m}$ which is additional evidence for the presence of a circumstellar disk.

Neither algorithm was able to find any periodic pattern for BZ Sgr using our data set.

\subsubsection{HBC $567=\mathrm{TW}$ CHA}

Previous periods of 8.6 and 8.0 days have been reported for TW Cha as well as a null result (see Table 2). TW Cha is a cTTS with a reportedly large degree of variability (Bouvier et al. 1986). This is confirmed here. In spite of the large amplitude, no convincing period-folded light curve presented itself. The reported periods of 8.0 and 8.6 days do not fold our data into a smooth light curve.

\subsection{7. $\mathrm{HBC} 570=\mathrm{CT} \mathrm{CHA}$}

We gather 10 entries in 1995 distributed over two months for CT Cha. It presents, in general, mild variability. The BSP algorithm and the DCDFT code indicate two periods with observations well distributed in phase and have large confidence levels: $9.86 \mathrm{~d}$ and 13.68d. Several other possible periods with acceptance larger than $90 \%$ cluster at about the former value. We tentatively suggest $9.86 \mathrm{~d}$ as the computed period for the season.

\subsubsection{HBC $591=\mathrm{T}$ CHA}

The pre Main Sequence nature of $\mathrm{T}$ Cha is established by Alcalá et al. (1993). In spite of an $\mathrm{EW}_{\mathrm{H} \alpha}$ typical of weak TTS, these authors report evidence of disk accretion - as inferred from the inverse P Cygni profile in the $\mathrm{H} \alpha$ - and robust spectral variability. We also have several medium resolution data targeting the $\mathrm{H} \alpha$ line that show strong variability (the PDS archives). The line goes from absorption to emission on a time scale of days, as is also reported in Alcalá et al. (1993).

Hoffmeister (1965) made extensive visual observations and proposed a period of 3.2436 days. A periodicity of $3.2 \mathrm{~d}$ is also found by Mauder \& Sosna (1975), although not confirmed by the more recent observations of Covino et al. (1992). This star consistently undergoes large photometric 
and spectroscopic variability (Covino et al. 1992; Alcalá et al. 1993).

Neither the BSP nor the DCDFT method reveal any conclusive period for our modest sample. Due in part to the variability revealed in the medium resolution data, we re-analyzed the data sets of Hoffmeister (1965) and Mauder \& Sosna (1975) using the DCDFT method. We averaged their data into a maximum of 3 data points per night. Hoffmeister's observations give a period of 3.221 days with a false alarm probability of $10^{-8}$. No period was found for the Mauder \& Sosna data set unless the observations taken after JD $=244141399$ are omitted, in which case we are able to reproduce the period of 3.22 days. Our 7-exposure light curve does not fold smoothly into a 3.22 day period.

We hypothesize that $\mathrm{T}$ Cha is another case of a TTS that undergoes phases of periodical behavior (see for example Vrba et al. 1989) and phases of irregular variations.

\subsubsection{HBC $590=\mathrm{SZ} 45$ and HBC $620=\mathrm{SZ} 108$}

These two stars are cTTS that show mild to low variability. Because of weather conditions we gathered a maximum of 10 points distributed over two consecutive years.

For SZ 45, the BSP periodogram method indicates two periods at 14.12 days and 14.75 days with comparably low $\chi^{2}$ 's. The period-folded light curves, however, are not well distributed in phase. The DCDFT method computes several possibilities, all clustering about a period of 13.00 days. We suggest the latter as a tentative value.

For SZ 108, both methods indicate similar solutions at about $4.05 \mathrm{~d}$ with a high level of confidence. However, the data are not well distributed in phase and due to the modest number of entries we regard the result as tentative.

\subsubsection{0. $\mathrm{HBC} 603=\mathrm{SZ} 77$}

SZ 77 shows a moderate range of variability in the course of our campaign (Table 1). Analysis of the near infrared excess indicates active disk accretion in this object (Batalha \& Basri 1993). Neither methods provide definitive period determinations.

\subsubsection{HBC $605=\mathrm{SZ} 82$}

SZ 82 was monitored by Covino et al. (1992) and no periodicity was found. It has signatures of disk accretion, with a mild near infrared excess indicative of reprocessed stellar light by overlying circumstellar dust. The ratio between the stellar and systemic luminosities tends to reinforce the presence of an active circumstellar disk (Batalha \& Basri 1993).

Two possible periods are indicated by the BSP: $1.16 \mathrm{~d}$ and 1.61d. The DCDFT method does not indicate a conclusive solution for the $V$-band, nevertheless two maxima in the power spectra are achieved at $7.42 \mathrm{~d}$ and $1.55 \mathrm{~d}$, both aliases. The confidence of both periods are $78 \%$ which leads us to conclude that no periodicity was established for SZ 82.

Table 2. Published Data for the Program Stars and Computed Periods. In the first column, the PDS or HBC numbers are listed. These pre main sequence stars are classified in the second column as wTTS (w) or cTTS (c) if indications of an accretion disk are present or not. See text for details. $\mathrm{H} \alpha$ line strengths are indicated in the third column. Periods that are found in the literature and determined in this work are respectively listed next

\begin{tabular}{|c|c|c|c|c|}
\hline $\mathrm{PDS} / \mathrm{HBC}$ & $\mathrm{w} / \mathrm{c}$ & $\mathrm{H} \alpha(\AA)$ & Lit. $P_{\text {day }}$ & $P_{\text {day }}$ \\
\hline PDS 1 & $\mathrm{w}$ & $0.2^{1}$ & - & - \\
\hline 45 & $\mathrm{w}$ & $2.0^{1}$ & - & - \\
\hline 50 & $\mathrm{w}$ & $12.5 / 8.0^{1}$ & - & - \\
\hline 59 & $\mathrm{w}$ & $5.0^{1}$ & - & 11.73 \\
\hline 66 & $\mathrm{c}$ & $47^{1}$ & - & 5.75 \\
\hline 70 & $\mathrm{w}$ & $2.0^{1}$ & - & 5.10 \\
\hline 77 & $\mathrm{w}$ & $2.0^{1}$ & - & 2.50: \\
\hline 81 & $\mathrm{w}$ & $0.7^{1}$ & - & - \\
\hline 82 & $\mathrm{c} / \mathrm{w}$ & $29.0 / 9.0^{1}$ & - & - \\
\hline 83 & $\mathrm{w}$ & $10.0^{1}$ & - & 5.52 \\
\hline 89 & $\mathrm{w}$ & $2.2^{1}$ & - & - \\
\hline 99 & $\mathrm{w}$ & $5.0^{1}$ & - & - \\
\hline 101 & $\mathrm{c}$ & $52^{1}$ & - & - \\
\hline HBC 565 & $\mathrm{c}$ & $49^{2}$ & $7.6^{3}, 6.2^{4}, 6.1^{5,6}$ & 5.97 \\
\hline 567 & $\mathrm{c}$ & $26^{2}$ & $8.6^{3}, 8.0^{4}, \mathrm{NP}^{6}$ & $\mathrm{NP}$ \\
\hline 570 & $\mathrm{c}$ & $56^{2}$ & - & 9.86: \\
\hline 578 & $\mathrm{c}$ & $71^{2}$ & $7^{4}, 7.2^{3}$ & 2.56 \\
\hline 583 & c & $52^{2}$ & - & 5.34 \\
\hline 590 & $\mathrm{c}$ & $32^{2}$ & - & 13.00: \\
\hline 591 & c & $2.0^{1}$ & $3.24^{4,10}, \mathrm{NP}^{7}$ & - \\
\hline 603 & c & $17^{2}$ & - & NP \\
\hline 605 & $\mathrm{w}$ & $7.0^{2}$ & $\mathrm{NP}^{7}$ & NP \\
\hline 620 & $\mathrm{w}$ & $0.5^{2}$ & - & 4.05: \\
\hline 656 & $\mathrm{c}$ & $54^{8}$ & & $3.12, \mathrm{NP}$ \\
\hline 657 & c & $28^{8}$ & & 4.05 \\
\hline $663 / 664$ & $\mathrm{c}$ & $10^{2}$ & $5.20^{11}, 4.83^{9}$ & 5.15 \\
\hline
\end{tabular}

1) GHETAL92, 2) Herbig \& Bell 1988, 3) Kappelman \& Mauder 1981, 4) Mauder \& Sosna 1975, 5) Schaeffer 1983, 6) Bouvier et al. 1986, 7) Covino et al. 1992, 8) Torres CAO, private communication, 9) Torres et al. 1983 (see text), 10) Hoffmeister (1965), 11) Chugainov (1974).

\subsection{Stars with detected periodicity}

\subsubsection{PDS 59}

PDS 59 is situated close to T Cha and may have a faint companion at 12 arcsec. The $\mathrm{EW}_{\mathrm{Li}}(500 \mathrm{~m} \AA)$ and the EW $_{\mathrm{H} \alpha}$ (less than $10 \AA$ ) are typical of wTTS (GHETAL92).

We have a total of 10 data entries distributed over three consecutive years (1989-1991) which is enough to 
cast suspicion on any result. Nevertheless, we proceed with the analysis having in mind that wTTS's have, in general, stable spot distributions (Herbst et al. 1994). The BSP method indicates $14.07 \mathrm{~d}$ and $11.73 \mathrm{~d}$ as possible periods, with the former folded light curve indicating a double wave shape. The DCDFT method reveals a peak in the power spectrum at $11.73 \mathrm{~d}$ in all colors, except $U$, with a modest confidence level of $30 \%, 98 \%, 96 \%$ and $83 \%$ in the colors $B, V, R$ and $I$ respectively. The peak and formal acceptance are significantly raised if all colors are included simultaneously. We propose a tentative period of $11.73 \mathrm{~d}$ and the folded light curve is presented in Fig. 1.

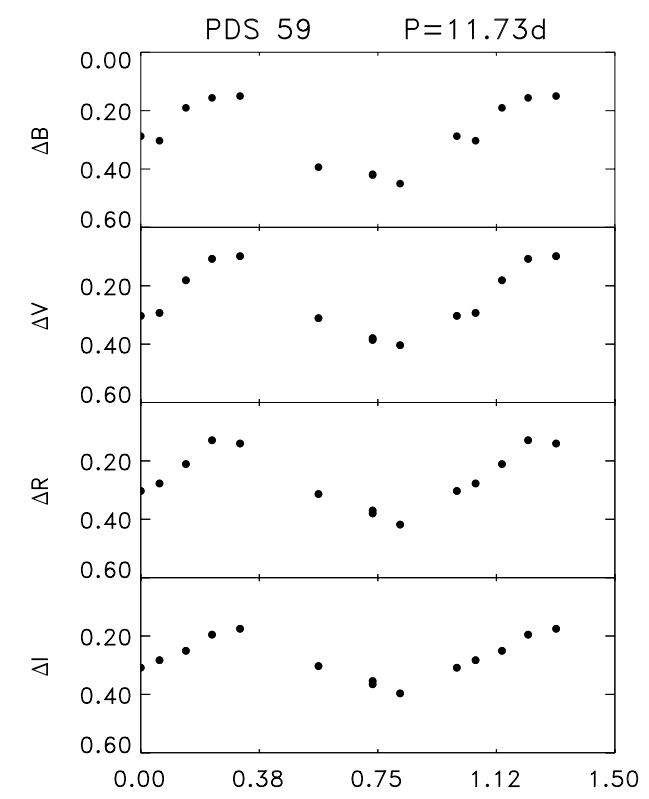

Fig. 1. Period-folded light curve for PDS 59

\subsubsection{PDS $66=$ Hen 892}

Hen 892 is a cTTS on the basis of its strong $\mathrm{H} \alpha, \mathrm{Li}$ $(370 \mathrm{~m} \AA)$ and IRAS colors. The large $\mathrm{EW}_{\mathrm{H} \alpha}$ indicates accretion which implies the presence of veiling and/or color excess, especially in the blue where these effects are most strongly felt. Nevertheless, the amplitude of variability of Hen 892 is merely $0.1 \mathrm{mag}$ in all colors. This variability is less than that found among active field stars (Rodonò 1986) and is atypical of cTTS. This object is isolated from any known star forming region.

We gathered a total of 9 entries in 1989/1990 (1/8). The BSP method indicates two acceptable solutions in the colors $V, R$ and $I$ : $4.67 \mathrm{~d}$ and $5.71 \mathrm{~d}$. The DCDFT method indicates $5.75 \pm 0.03$ days with a confidence of $97.2 \%$ and $4.70 \pm 0.02$ with a confidence of $93.5 \%$. The criteria to adopt 5.75 days as the period for the season is based on the shape and the phase coverage of the folded light curve (Fig. 2).

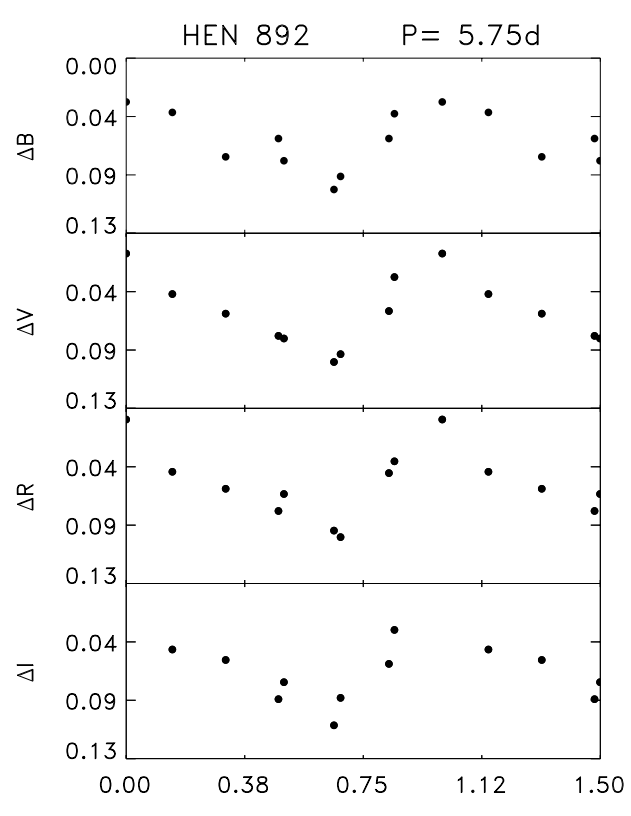

Fig. 2. The same as Fig. 1 for Hen 892

\subsubsection{PDS $70=$ CoD- 408434}

The $\mathrm{H} \alpha$ line of PDS 70 varies in between $2-4 \AA$. It shows a low range of variability ( 0.1$)$ which is independent of color.

We gathered 9 entries $U B V(R I)_{\mathrm{C}}$ during $1989 / 1990$ $(1 / 8)$. The BSP method fits the data well at $5.1 \mathrm{~d}$ or $5.6 \mathrm{~d}$, with comparable and low $\chi^{2}$ and good phase coverage. The power spectrum from the DCDFT method peaks at $5.1 \mathrm{~d}$ and $4.26 \mathrm{~d}$ with a large degree of confidence in the $V-$ band. The confidence of the latter value is not consistently reproduced in the other colors. Therefore, we suggest a period of 5.1d during these two years (Fig. 3).

\subsubsection{PDS $83=$ V896 Sco}

A steady increase in the amplitude towards the blue can be seen in the light curves of V896 Sco. Our data set is very modest with one entry taken in 1989 and six others in the following year. The periodogram analysis done with the BSP method indicates two possible periods at 5.54 days and 7.93 days yielding good phase coverage and low $\chi^{2}$ values. The DCDFT method indicates 5.52 days with a large confidence level after adding the magnitudes of all colors. Thus, inspection of both period-folded light curves leads us to suggest 5.52 days as the rotational period of V896 Sco (Fig. 4).

\subsection{5. $\mathrm{HBC} 565=\mathrm{SY}$ CHA}

SY Cha is an active $\mathrm{T}$ Tauri showing, at times, complex light curve behavior which suggests the presence of more than one dominant spot (Bouvier \& Bertout 1989) and changes in the spot distribution. Periodicities 


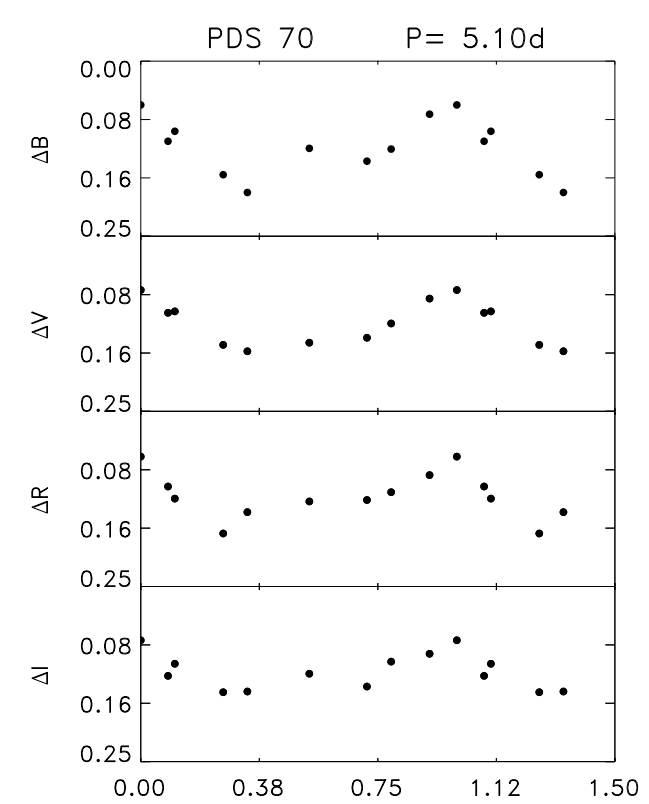

Fig. 3. The same as Fig. 1 for PDS 70

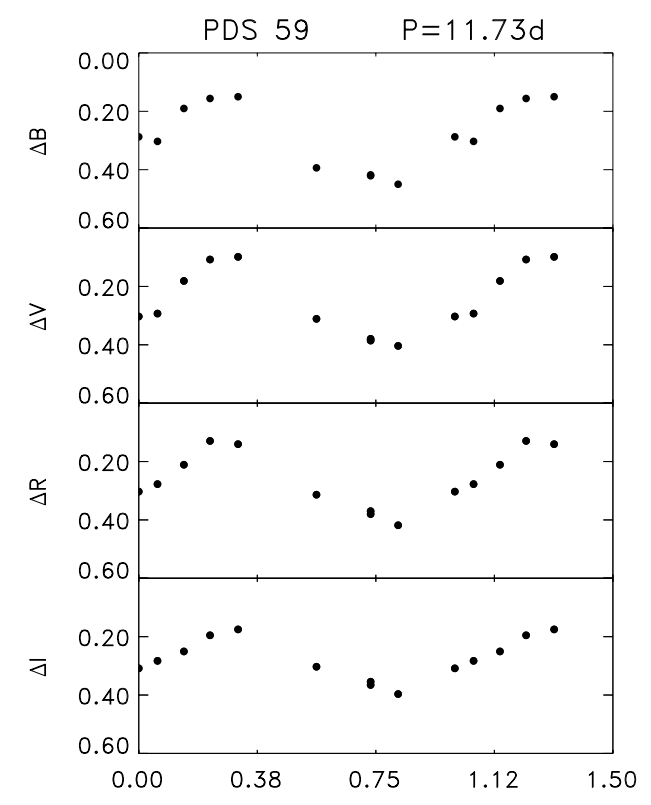

Fig. 4. The same as Fig. 1 for V896 Sco

have been reported for this object in Bouvier \& Bertout (1989); Schaeffer (1983); Kappelman \& Mauder (1981) and Mauder \& Sosna (1975). These authors report 6.0, $6.129,7.6$ and $6.2 \mathrm{~d}$, respectively.

We gathered a total of 22 observations during 1994 and 1995 (6/16) and we confirm the periodic behavior of SY Cha. The BSP algorithm indicates two periods at $5.97 \mathrm{~d}$ and $6.07 \mathrm{~d}$. The former gives more complete phase coverage. The DCDFT method suggests 5.97 days as the most acceptable period for the season with confidence levels of $99.99 \%$. We conclude that during our campaign SY Cha had a period of $5.97 \mathrm{~d}$ and the final folded light curve is presented in Fig. 5.

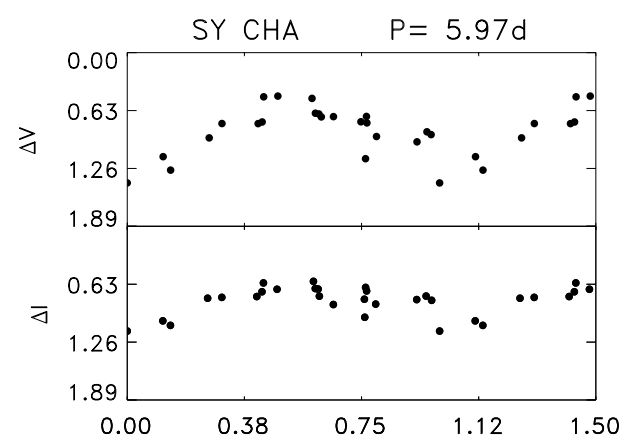

Fig. 5. The same as Fig. 1 for SY Cha

\subsection{6. $\mathrm{HBC} 578=\mathrm{VZ}$ CHA}

VZ Cha is a typical representative of cTTS, with very large $\mathrm{EW}_{\mathrm{H} \alpha}$ and IRAS fluxes. Studies based on spectroscopic observations indicate a period of about 7 days (Mauder \& Schulz 1978). Another period of 7.2 days - determined with sparser time sampling - is also pointed out by Kappelman \& Mauder (1981).

The BSP method does not indicate any significant minimum. The DCDFT algorithm presents peaks in the power spectrum at 2.577 days $(99.8 \%$ in $I$ and $98.0 \%$ in $V$ ), and, with lower significance, 12.00 days. We suggest 2.56 days as the final period, after inspecting the periodfolded light curve (Fig. 6).

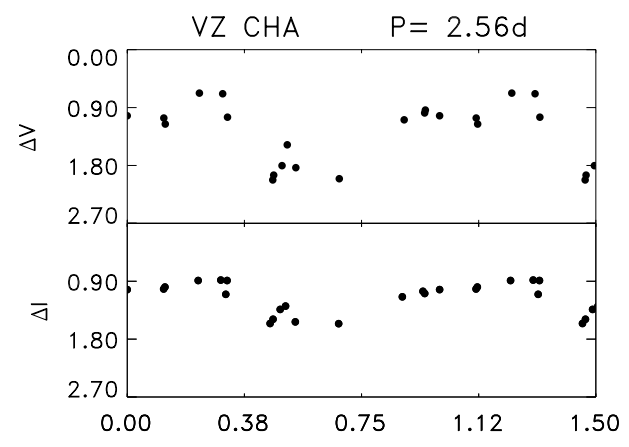

Fig. 6. The same as Fig. 1 for VZ Cha

\subsubsection{HBC $583=$ WY CHA}

We have photometry of WY Cha taken during two seasons. The BSP method indicates two acceptable periods of $5.43 \mathrm{~d}$ and $2.10 \mathrm{~d}$, with the former showing better phase coverage. The DCDFT method indicates the period of 5.34 
days with a very high level of acceptance. The former period gives the minimum $\chi^{2}$ for the adjusted curve and is chosen as the period for the season (Fig. 7).

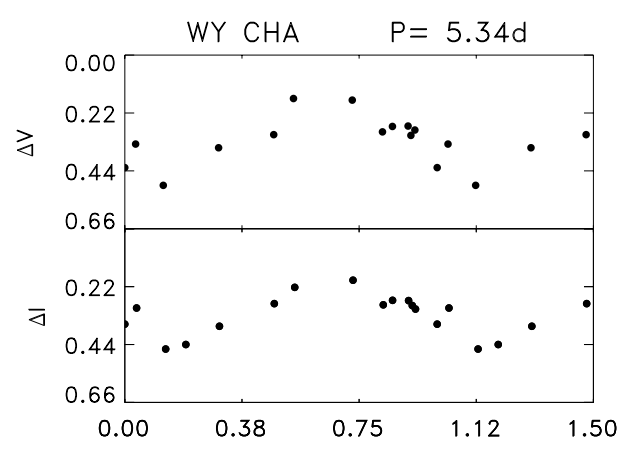

Fig. 7. The same as Fig. 1 for WY Cha

\subsubsection{HBC $656=$ AS 216}

Our photometric data of AS 216 - a PMS star not associated with any known molecular cloud (Quast et al. 1987 ) - have been gathered since 1985. The $\mathrm{EW}_{\mathrm{H} \alpha}$ computed at medium resolution (GHETAL92) spans values between 40 and $70 \AA$ which is indicative of disk-like activity. The photometric variability of AS 216 shows no significant change in the first three years. It reaches an amplitude of $0.15 \mathrm{mag}$ in the $V$-band during the interval JD $=2446223-2446279$, rises up to $0.25 \mathrm{mag}$ during $\mathrm{JD}=2446627-2446648$, and then decreases again to 0.15 mag ( JD $=2446978-2447028)$. This behavior is consistent for all the colors. In the last two years $(1989 / 1990)$ AS 216 went through an overall increase in variability, which is best observed in the $U$ band.

Both algorithms indicate the periods $3.12 \mathrm{~d}$ and $4.24 \mathrm{~d}$ during the first three runs, independent of whether the data sets are used together or separately in the periodogram analysis. A slightly larger confidence is given to the $3.12 \mathrm{~d}$ period leading us to adopt it as the photometric period of 1985/1986/1987. However, neither of these options reaches the minimum level of acceptance in the following two years $(1989 / 1990)$. In fact, the BSP method gives a period of $6.69 \mathrm{~d}$ which is not confirmed by the DCDFT method, in part because the period-folded light curve shape departs slightly from a sinusoidal one. We conclude that AS 216 is another case of a cTTS that undergoes periodical/aperiodical phases.

The folded light curve of the 3.12 day period $(1985 / 1986 / 1987)$ is shown in Fig. 8. We note the occurrence of a maximum in brightness at phase 0.85 that is detected in the three colors. It is not an artifact of data reduction nor weather conditions. In fact, it indicates a significant increase of continuum emission which is not compatible with classical flare activity but with the optical veiling detected in PMS stars (Basri \& Batalha 1990).
We show in Fig. 9 the 1989/1990 photometry, folded into 3.12 days.

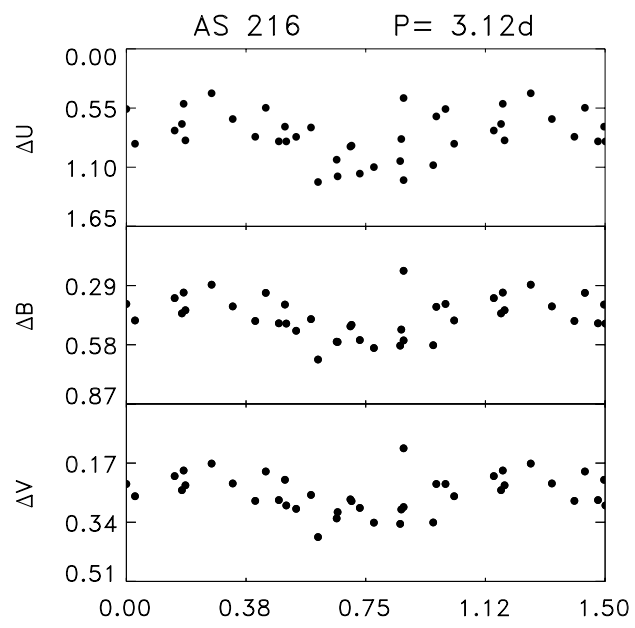

Fig. 8. $U B V$ period-folded light curve for AS 216 during 1985 , 1986 and 1987 observing season

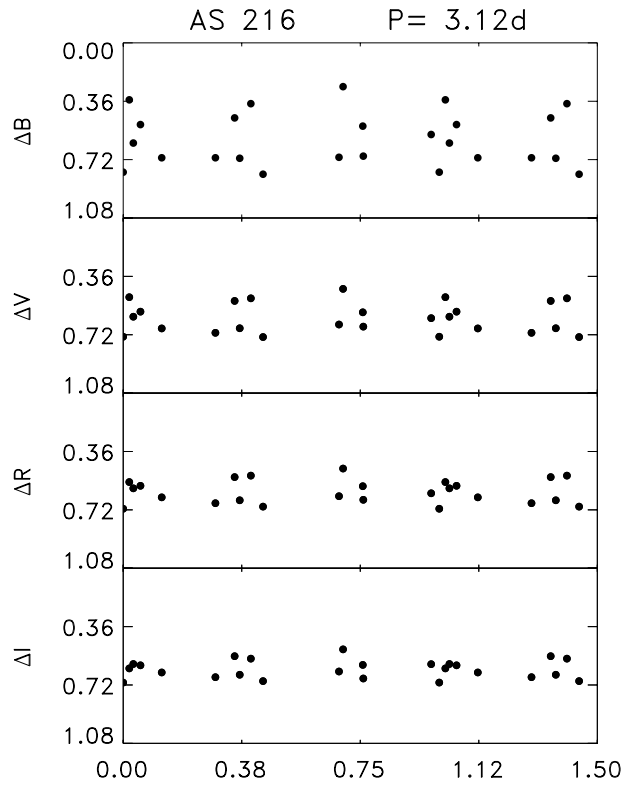

Fig. 9. The 3.12 days period-folded light curve of AS 216 during the 1989/1990 season. Inspection in the Fig. 8 show that AS 216 is a typical case of cTTS that goes through phases of periodical/aperiodical behavior.

\subsection{9. $\mathrm{HBC} 657=\mathrm{AS} 218$}

AS 218 has shown evidence of periodic or quasi-periodic modulation in several colors with possibly no connection to cold spots (Quast et al. 1987). It is also shown that this 
object is isolated from any molecular cloud (Quast et al. 1987).

The BSP method indicates periods of less than 2.0 days which are not included in our final analysis. The folded light curve with a period of 4.05 days obtained by the DCDFT method is indicated in Fig. 10.

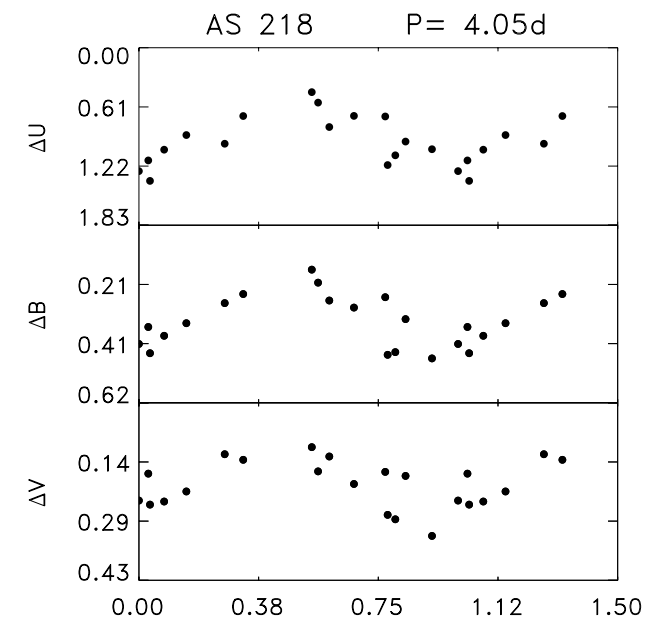

Fig. 10. The same as Fig. 1 for AS 218

\subsubsection{HBC 663/664 = FK Ser}

FK Ser is classified as a post T Tauri Star by Herbig (1973) and a visual binary system with separation of $1.33^{\prime \prime}$ (HBC $663 / 664)$. It presents $800 \mu \mathrm{m}$ emission similar to that of PDS 55, and Jensen et al. (1996) conclude that FK Ser has disk properties similar to that of a typical TTS.

Quast et al. (1987) suggest that this system is isolated from any known molecular cloud, and they note that the periodic modulation is incompatible with those of cool spots. Chugainov (1974) finds a sinusoidal light variation with a period of 5.20 days and an amplitude of 0.1 magnitudes. Observations of this star in $B$ and $V$ done in 1974 at CTIO result in a period of 4.83 days, erroneously printed as 4.53 days in Torres et al. (1983). The origin of the apparent discrepancy in the resulting periods may reside in the fact that FK Ser is a binary system. Each component has a similar spectral classification. Therefore, the continuum modulation of each component, if present, will necessarily add to the final light curve explaining the difference in periods.

We have 30 entries taken during 10 nights in the 1986 run $U B V$ and 17 taken during the 1988-1990 one $U B V(R I)_{\mathrm{C}}$. In $1986 \mathrm{FK}$ Ser shows large amplitude variations - a maximum in $U$. The period of $4.89 \mathrm{~d}$ presents itself as the best solution of the BSP algorithm for both runs. The DCDFT method finds a period of $5.13 \mathrm{~d}$ in the first run if the photometry of all colors are added simultaneously. The variability is conspicuous in the following run, but the indicated period of $5.15 \mathrm{~d}$ has a lower confidence level than for the previous run. We suggest a photometric period of 5.15d for FK Ser (Fig. 11).

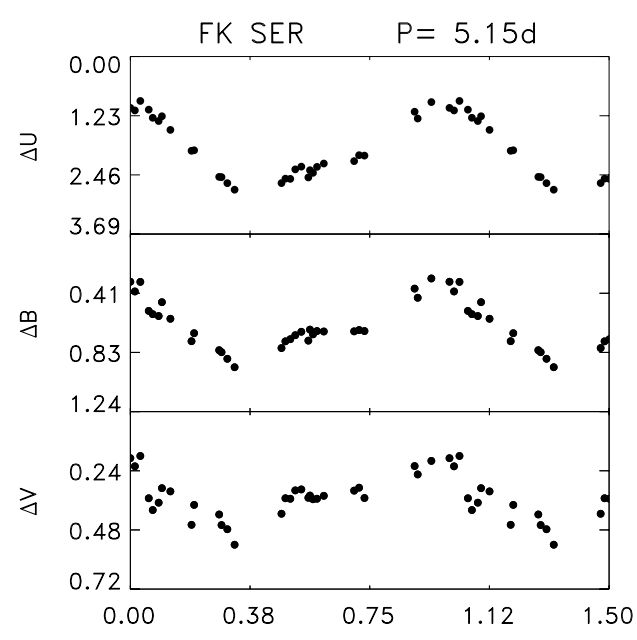

Fig. 11. The same as Fig. 1 for FK Ser

\section{Summary and conclusions}

We present the photometric variability of $26 \mathrm{~T}$ Tauri Stars in an effort to increase the statistics of the photometric data of pre-Main Sequence Stars and to deepen the information regarding TTS observed in the Southern hemisphere. More than half of our objects were initially identified using IRAS data and subsequently classified using medium resolution spectra revealing the LiI $\lambda 6707$ line in absorption together with the $\mathrm{H} \alpha$ line in emission or filled in (GHETAL92). The other objects in our sample are listed in the HBC catalogue.

We derive photometric periods for PDS 59, Hen 892, PDS 70, V896 Sco, VZ Cha, WY Cha, AS 216, AS 218 and FK Ser. We suggest possible periodicity during the observing season for PDS 77, CT Cha, SZ 45, SZ 108 and confirm previous values for SY Cha. Irregular variability is found for SZ 77, SZ 82 and TW Cha.

TW Cha shows no periodicity during the observing season, in agreement with the previous work of Bouvier et al. (1986). It is worth noting that Kappelman \& Mauder (1981) and Mauder \& Sosna (1975) do find periodic patterns in the TW Cha light curve. Therefore, TW Cha should be regarded as an example of a cTTS whose light curve may be either rotationally modulated or dominated by irregular variations. Likewise, our exercise on the archived data of T Cha and our analysis of AS 216 produce similar results - results which suggest these stars have rotational modulations which are occasionally dominated by irregular flux variations.

The rich time series of AS 216 distributed over 5 years allows us to test the stability the measured rotational 
period. The light curve modulates with a period of $3.12 \mathrm{~d}$ between 1985 and 1987. This result is obtained whether the entire data set is taken together or each year is considered separately. During the first three years, the amplitude is larger in the bluer bands. The light curve amplitudes as a function of wavelength reveal that a hot spot is likely controlling the periodic modulation. Based on the persistence of the $3.12 \mathrm{~d}$ period, the spot is presumably stable. In subsequent observing seasons (1989-1990), we are not able to measure any periodicity. Instead, we find larger amplitude, irregular variations.

Acknowledgements. We would like to express our appreciation to Dr. C. Akerlof for helping us to install the cubic $B$-spline code and for showing us how to use it. We thank the referee Dr. E. Covino, for a careful and critical reading of the paper, leading to an improved final version.

\section{References}

Akerlof C., Alcock C., Allsman R., et al., 1994, ApJ 436, 787

Alcalá J.M., Covino E., Franchini M., et al., 1993, A\&A 272, 225

Basri G., Batalha C.C., 1990, ApJ 363, 654

Batalha C.C., Basri G., 1993, ApJ 412, 363

Bouvier J., Wichmann R., Grankin K., et al., 1997, A\&A 318, 495

Bouvier J., Covino E., Kovo O., et al., 1995, A\&A 299, 89

Bouvier J., Cabrit S., Fernandez M., Martin E.L., Matthews, J.M., 1993, A\&A 272, 176

Bouvier J., Bertout C., 1989, A\&A 211, 99

Bouvier J., Bertout C., Benz W., Mayor M., 1986, A\&A 165, 110

Chugainov P.F., 1974, IzKry 32, 3C

Covino E., Terranegra L., Franchini M., Chavarria K,. Stalio R., 1992, A\&AS 94, 273

de la Reza J.R., Torres C.A.O., Quast G., Castilho B.V., Vieira G.L., 1989, ApJ 343, L61

Edwards S., Hartigan P., Ghandour L., Andrulis C., 1994, AJ 108,1056

Edwards S., Strom S.E., Hartigan P., et al., 1993, AJ 106, 372

Ferraz-Mello S., 1981, AJ 86, 619

Ferraz-Mello S., Quast G.R., 1987, Exercises in Astronomy, Kleczek J. (ed.). D. Reidel Pub. Co.

Gahm G.F., Gullbring E., Fischerstrom C., Lindroos K.P.,
Loden K., 1993, A\&AS 100, 371

Gregório-Hetem J., Lépine J.R.D., Quast G.R., Torres C.A.O., de la Reza J.R., 1992, AJ 103, 549 (GHETAL92)

Hartmann L., Hewett R., Calvet N., 1994, ApJ 426, 669

Herbig G.H., 1973, ApJ 182, 129

Herbig G.H., Bell K.R., 1988, Lick Obs. Bull. No. 1111

Herbst W., Herbst D.K., Grossman E.J., Weinstein D., 1994, AJ 108, 1906

Hoffmeister C., 1965, Veröffentlichungen der Sternwarte in Sonneberg Band 6 Heft 3, 97

Jablonski F., Baptista R., Barroso Jr. J., Gneiding C.D., Rodrigues F., Campos R.P., 1994, PASP 106, 1172

Joy A.H., 1942, PASP 54, 15

Johns C.M., Basri G., 1995, AJ 109, 2800

Joncour I., Bertout C., Menard F., 1994, A\&A 285, L25

Kappelman N., Mauder H., 1981, ESO Messenger 23, 18

Kenyon S.J., Hartmann L., Hewett R., Carrasco L., CruzGonzales I., Recillas E., Salas L., Serrano A., Strom K.M., Strom S.E., 1994, AJ 107, 2153

Königl A., 1991, ApJ 370, L39

Magnani L., Blitz L., Mundy L., 1985, ApJ 295, 402

Marraco H.G., Rydgren A.E., 1981, AJ 86, 82

Mauder H., Sosna F.M., 1975, Inf. Bull. Var. Stars 1049

Mauder H., Schulz E., 1978, Mitteil. Astron. Gesellschaft 43, 181

Quast G.R., Torres, C.A.O., de la Reza J.R., Mello G.F.P., 1987, Rev. Mex. Astron. Astrofis 14, 360

Rodonò M., 1986, In Highlights of Astronomy. Vol. 7 - Proc. of the $9^{\text {th }}$ IAU Gen. Assembl., Delhi, India. Dordrecht, D. Reidel Publ. Co.

Schaeffer B.E., 1983, ApJ 266, L45

Shu F.H., Najita J., Ostriker E., Wilkin F., Ruden S., Lizano S., 1994, ApJ 429, 781

Smith M.D., 1994, A\&A 287, 523

Stephenson C.B., Sanduleak N., 1977, ApJS 33, 459

Strassmeier K.G., Welty A.D., Rice J.B., 1994, A\&A 285, L17

Torres C.A.O., Busko I.C., Quast G.R., 1983, IAU Coll. 71, 175

Torres C.A.O., Quast G.R., de la Reza J.R., 1987, Rev. Mex. Astrofis. 14, 361

Torres C.A.O., Quast G.R., de la Reza J.R., Gregório-Hetem J., Lépine J.R.D., 1995, AJ 109, 2146

Vrba F.J., Rydgren A.E., Chugainov P.F., Shakovskaya N.I., Weaver W.B., 1989, AJ 97, 483 